\section{Schmerzgedächtnis und Sensibilisierung}

Dieter Kleinböhl, Dagmar Baus, Ulrike Hornberger, Rupert Hölzl

Labor für Klinische Psychophysiologie, Otto-Selz-Institut, Universität Mannheim

psychoneuro 2005; 31 (2): 84-91

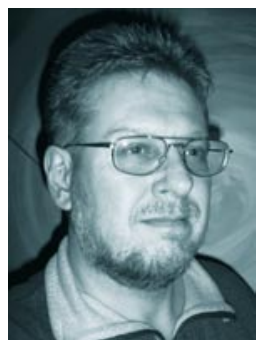

Dieter Kleinböhl

Bei chronisch Schmerzkranken findet man häufig eine Zunahme der subjektiven Schmerzempfindung bei konstanter anhaltender oder wiederholter Reizung mit experimentellen Schmerzreizen, die zum Zweck der psychophysikalischen Schmerzdiagnostik appliziert werden. Eine solche Schmerzsensibilisierung wird als wahrnehmbares Korrelat neuronaler Plastizität auf frühen peripheren und spinalen Schmerzverarbeitungsstufen betrachtet und ist möglicherweise ein Indikator für eine im Laufe der Chronifizierung langfristig veränderte afferente Schmerzverarbeitung. Schmerzsensibilisierung wird aber nicht nur durch neuroplastische Prozesse, die ohne höhere zerebrale Verarbeitung auskommen, moduliert, sondern auch durch zerebral lokalisierte Lernprozesse, wie der klassischen und operanten Konditionierung. Operantes Lernen zunehmender Sensibilisierung in Verbindung mit dem psychologischen Mechanismus der Furcht-Vermeidung ist möglicherweise eine zentrale Ursache der Schmerzchronifizierung bei Schmerzsyndromen mit unklarer Genese, vor allem bei den häufigen Schmerzsyndromen mit Beeinträchtigung des muskuloskelettalen Systems.

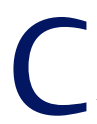
hronische Schmerzen des muskuloskelettalen Systems sind in westlichen Industrienationen weit verbreitet und verursachen einen Großteil der Kosten im Gesundheitswesen. Bei Rückenschmerzen beträgt die Prävalenz in diesen Ländern 60-80\% und die durch Chronifizierung des Schmerzes bedingte Invalidität nimmt über die Zeit rasch zu. Demgegenüber sind Ätiologie und Chronifizierungsmechanismen muskuloskelettaler Schmerzen nur unzureichend aufgeklärt. Häufig sind auslösende Noxen oder organische Ursachen bei diesen Patienten nicht nachweisbar, weshalb von „funktionellen“ Schmerzen oder im Falle des Rückenschmerzes von „unspezifischen“ Rückenschmerzen gesprochen wird. Als Ursache für die Entstehung und
}

Chronifizierung muskuloskelettaler Schmerzen werden derzeit neurobiologische Prozesse angenommen, welche unter dem Eindruck einer auslösenden Noxe oder früheren Schmerzerfahrungen eine dauerhafte Veränderung der nozizeptiven Signalübertragung im Nervensystem bewirken (10).

Solche funktionellen und strukturellen Veränderungen der schmerzverarbeitenden Strukturen im Nervensystem („Pain-Neuromatrix“) werden als „Schmerzgedächtnis“ oder
„Schmerzspur“ bezeichnet. Unter diesem weit gefassten Oberbegriff werden von Seiten der Physiologie neurobiologische Prozesse vor allem auf der Ebene des Rückenmarks verstanden, wie zum Beispiel die zentrale Sensitivierung nozizeptiver Neurone des Hinterhorns durch Langzeitpotenzierung und andere Prozesse neuronaler Plastizität (vgl. Beitrag von Sandkühler in dieser Ausgabe). In der Psychologie sind dagegen kognitive und an eine zerebrale Verarbeitung gebundene Lernprozesse wie Sensibilisierung, Habituation, operante und klassische Konditionierung gemeint, sowie das an eine frühe Verarbeitungsstufe in der Wahrnehmung geknüpfte „priming“ (vgl. Beitrag von Flor in dieser Ausgabe). Die dem Schmerzgedächtnis zugrunde liegenden Lernprozesse und neuroplastischen Veränderungen sind unabhängig von spinaler oder zerebraler Lokalisation dem bewussten Erleben nicht zugänglich, verändern aber ihrerseits Wahrnehmung und Verhalten, weshalb man auch vom „impliziten Schmerzgedächtnis“ spricht.
Gefördert durch die Deutsche Forschungsgemeinschaft: Klinische Forschergruppe 107 „Neuronale Plastizität und Lernprozesse in der Schmerzchronifizierung“, Projekt Ho 904/11-(1-4) sowie durch die Berufsgenossenschaft Nahrungsmittel \& Gaststätten Mannheim, Geschäftsbereich Prävention. 
I

Ist das Schmerzgedächtnis messbar?

Die durch das implizite Schmerzgedächtnis verursachten Veränderungen von Wahrnehmung und Verhalten sind die Grundlage diagnostischer Verfahren zum Nachbeim chronischen Schmerz mutmaßlich veränderten Verarbeitung nozizeptiver Signale im Nervensystem. In der klinischen Schmerzdiagnostik und in der Schmerzforschung werden deshalb Methoden und Verfahren der Psychophysik (einem Teilgebiet der Psychophysiologie) eingesetzt, bei denen unter kontrollierten Reizbedingungen die durch das Schmerzgedächtnis verursachten spezifischen Wahrnehmungsund Verhaltensänderungen in einem Laborkontext erzeugt und gemessen werden können. Klinische Schmerzen sind im Sinne eines auslösenden weis und zur Charakterisierung der physikalischen Reizes nicht definiert und deshalb als Ausgangspunkt für eine psychophysikalische Funktionsprüfung ungeeignet. Man bedient sich deshalb so genannter experimenteller Reizmodelle, bei denen in unterschiedlichen Sinnesmodalitäten physikalisch definierte Reize nicht-schmerzhafter und schmerzhafter Qualität zur Prüfung von Verarbeitungsfunktionen im Nervensystem verwendet werden. Diese Reiztechniken wurden meist in der tierexperimentellen Schmerzforschung entwickelt und später auf den Menschen übertragen. Es werden aufgrund ihrer einfachen Anwendbarkeit und Ungefährlichkeit für den Patienten überwiegend thermische, mechanische und elektrische Reizung der Haut oder der Muskulatur angewendet. Obwohl bei diesen Reizmodi auch schmerzhafte Reizstärken appliziert werden,

\section{Abb. 1 Psychophysikalisches Verfahren zur Messung der Sensibilisierung bei tonischer Hitzereizung}

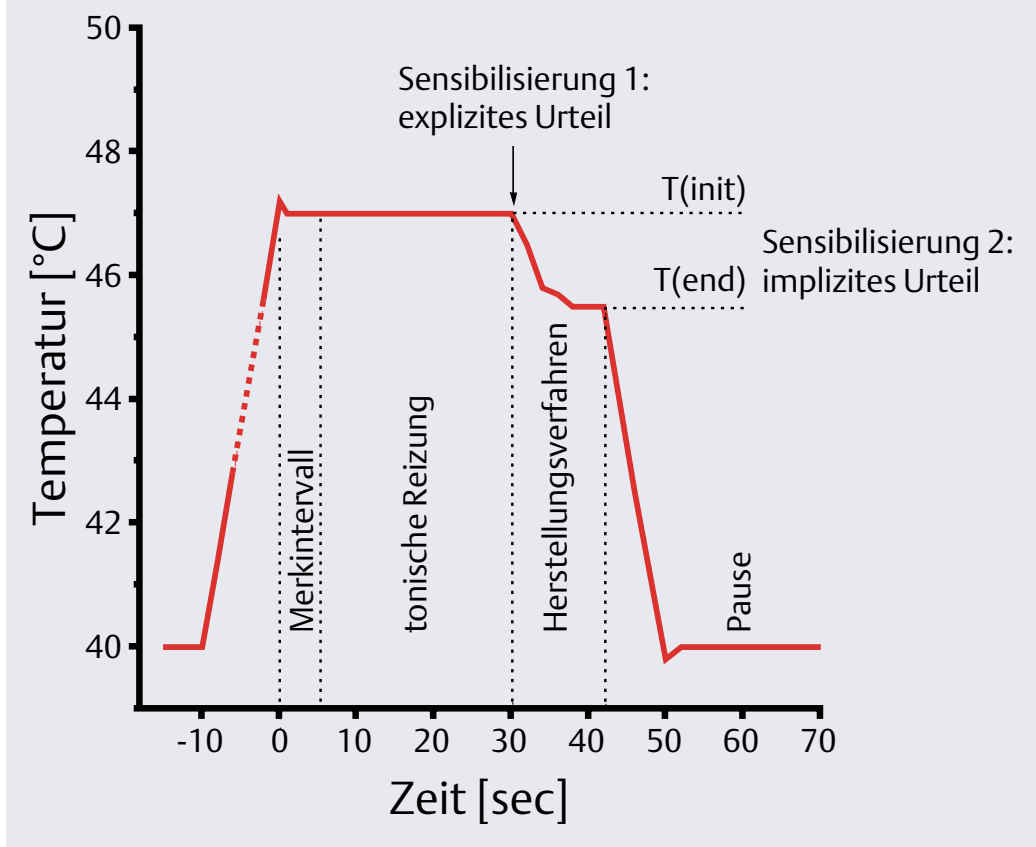

Beim Verfahren der „dualen Sensibilisierung“ (6) wird die subjektive Empfindungsänderung im Verlauf konstanter tonischer Hitzereize von 30 Sekunden Dauer erfasst. Auf einer Schätzskala wird die Größe der subjektiven Empfindungsänderung im Vergleich zur anfänglichen Empfindungsstärke direkt eingeschätzt (explizites Urteil). Anschließend stellt der Proband in einem Herstellungsverfahren eine Temperatur ein, welche der initialen Empfindung im Merkintervall entspricht. Die Differenz von Anfangs- und Endtemperatur ergibt das zweite, implizite Sensibilisierungsmaß. Die thermische Kontaktreizung wird mit Thermoden auf der Haut, beispielsweise am Thenar der Hand, durchgeführt. sind die Reize gut verträglich, wiederholbar und werden, bei entsprechender Instruktion, vom Patienten gut toleriert. Bei den angewendeten psychophysikalischen Verfahren werden somatosensorische Funktionsprüfungen angewendet, bei denen beispielsweise Schwellen für die Wahrnehmung eines Reizes (war der Reiz spürbar?) oder für bestimmte Empfindungen (war der Reiz schmerzhaft?) gemessen werden. Solche Verfahren werden auch unter dem Oberbegriff der „quantitativen sensorischen Testung“ (QST) zusammengefasst. Darüber hinaus werden spezielle Reizmodelle mit wiederholter (repetitiver) oder anhaltender (tonischer) Reizung eingesetzt, die dazu geeignet sind, dynamische Veränderungen in der Reizverarbeitung $\mathrm{zu}$ induzieren und gleichzeitig zu messen (Abb. 1). Die Zielsetzung bei der Anwendung dieser Modelle besteht nicht darin, klinische Schmerzen möglichst genau zu modellieren, sondern Aspekte der Reizverarbeitung messbar zu machen, die etwas über das implizite Schmerzgedächtnis aussagen können, wie Sensibilisierung oder Habituation (Abb. 1). Welche Befunde liefern solche Verfahren bei chronisch Schmerzkranken im Vergleich zu Gesunden?

\section{Sensibilisierung bei chro- nischen Schmerzsyndromen}

Untersucht man bei chronisch schmerzkranken Patienten den Aspekt der dynamischen Veränderung der Schmerzwahrnehmung bei wiederholter oder anhaltender Reizung mit konstanter Reizstärke, findet man vor allem bei Patienten mit muskuloskelettalen Beschwerden eine gegenüber Gesunden verstärkte Sensibilisierung (vgl. die Übersicht bei 2). Unsere eigenen Arbeiten mit tonischer Kontakthitzereizung als Reizmodell (Abb. 1) zeigen verstärkte Sensibilisierung bei Patienten mit unspezifischen Rückenschmerzen (Abb. 2) und nach neueren Daten finden wir dies in noch stärkerem Maße auch beim Fibromyalgiesyndrom (6, 7). Mit wiederholter Strahlungshitzereizung als Reizmodell wurde ebenfalls verstärkte Sensibilisierung bei unspezifischen Rückenschmerzen, Fibromyal- 



Die subjektive Sensibilisierung wird als scheinbare Intensitätszunahme von konstanten tonischen Hitzereizen im Verlauf von 30 Sekunden Reizung erfasst (zeitliche Summation; vgl. Abb. 1). Während Gesunde bei nicht-schmerzhaften und bei schmerzhaften Reizstärken Habituation, d.h. eine Abnahme der subjektiven Empfindungsstärke, zeigen, erleben chronisch Rückenschmerzkranke schon bei nicht ,,schmerzhaften Reizstärken Sensibilisierung, d.h. eine Zunahme der Empfindungsstärke, die bei schmerzhaften Reizstärken noch zunimmt (nach 6).

gie (12) und darüber hinaus bei temporomandibulären Schmerzen gefunden (9). Verstärkte Sensibilisierung beim chronischen Schmerz ist außerdem nicht auf muskuloskelettale Beschwerden beschränkt (2). Unsere eigenen Daten zeigen dies bei thermischer tonischer Reizung auch bei Patienten mit chronischen episodischen Kopfschmerzen (Spannungskopfschmerz und Migräne), wenn auch nicht in so starkem Ausmaß wie bei unspezifischen Rückenschmerzen (6). Für Migräne mit und ohne Aura konnte dieser Befund bei thermischer Reizung nicht bestätigt werden, allerdings wurde bei diesen Patienten verstärkte Sensibilisierung bei repetitiver elektrischer und mechanischer Reizung gefunden (14). Verschiedene neuropathische Schmerzen gehen ebenfalls mit verstärkter Sensibilisierung einher (2).

Neurobiologische Modelle

Erhöhte Schmerzempfindlichkeit und verstärkte Sensibilisie- rung in der Wahrnehmung wiederholter oder anhaltender Schmerzreize ist nach diesen Befunden ein häufiges klinisches Merkmal, vor allem bei chronischen muskuloskelettalen Schmerzen. Es stellt sich jedoch die Frage, in welcher Beziehung diese Merkmale zum impliziten Schmerzgedächtnis und zur Schmerzchronifizierung stehen?

\section{Sensibilisierung, zeitliche} Summation und windup

Wiederholte schmerzhafte Reizung mit drei oder mehr kurzen Reizen pro Sekunde, aber auch anhaltende tonische Schmerzreize führen zu einem Prozess aktivierungsabhängiger Neuroplastizität an multirezeptiven Neuronen im Hinterhorn des Rückenmarks. Die Freisetzung von Glutamat und die anhaltende nozizeptive Erregung öffnen auf diesen Neuronen Ionenkanäle mit N-Methyl-D-aspartat (NMDA)-Rezeptoren, wodurch Kalzium in die Zelle einströmt und durch beschleunigte Depolarisation der Zellmembran eine kurzfristige und reversible Empfindlichkeitssteigerung des nozizeptiven Neurons bewirkt (vgl. Beitrag von Sandkühler in dieser Ausgabe). Im Zeitbereich unterhalb einer Minute führt dies zu einer dynamischen Zunahme der neuronalen Reaktion bei konstanter anhaltender oder wiederholter Reizung (engl. „windup“). Dieser aus Tierexperimenten bekannte und gut untersuchte Prozess hat im Humanmodell in der subjektiven Wahrnehmung eine Entsprechung in einer Zunahme der subjektiven Empfindungsstärke, also einer Sensibilisierung bei wiederholter oder anhaltender Reizung mit konstanter Reizstärke. In der Literatur wird dies auch als zeitliche Summation (engl. „temporal summation“) bezeichnet, nach dem früher als Ursache vermuteten zugrunde liegenden neuronalen Prozess. Der windup nozizeptiver Hinterhornneurone spielt sicherlich bei der den klinischen Befunden zugrunde liegenden kurzfristigen Sensibilisierung im Bereich unterhalb einer Minute eine Rolle, wie pharmakologische Blockaden des NMDA-Rezeptors gezeigt haben: analog zur Blockade des windup im Tierexperiment kann im Humanversuch die subjektive Sensibilisierung durch Gabe von NMDAAntagonisten wie Ketamin, Amantadinsulfat oder Memantine verhindert werden. Dies wurde mehrfach für verschiedene Reizmodelle, die subjektive Sensibilisierung auslösen, gezeigt (2). Ob aber eine Blockade der verstärkten Sensibilisierung bei den diskutierten Schmerzsyndromen mit NMDA-Antagonisten möglich ist und dies gleichzeitig mit einer Besserung des klinischen Schmerzes einhergeht, ist derzeit weitgehend ungeklärt. Eigene Studien scheinen einen solchen Zusammenhang zu belegen, dies muss aber durch größere Untersuchungsstichproben bestätigt werden.

\section{Sekundäre Hyperalgesie und zentrale Sensitivierung}

Der zweite neurobiologische Mechanismus des Schmerzgedächtnisses, der aus dem Tierexperiment in die Humanforschung übertragen werden konnte, ist die zentrale Sensitivierung nozizeptiver afferenter Rückenmarksneurone durch Langzeitpotenzierung (vgl. Beitrag von Sandkühler in diesem Heft). Diese Empfindlichkeitssteigerung wird durch eine hochfrequent repetitive elektrische Reizung ausgelöst und verändert für Stunden nach dem konditionierenden Reiz die Verarbeitung im angrenzenden Hautareal für mechanische Reize (Allodynie) und die Schmerzverarbeitung (sekundäre Hyperalgesie; vgl. 4). Zentrale Sensitivierung ist deshalb eher als der reversible windup geeignet, eine zeitlich andauernde erhöhte Schmerzempfindlichkeit zu erklären und darf keinesfalls, wie häufig in der Literatur geschehen, mit windup gleichgesetzt werden. Allerdings sind die Effekte zentraler Sensitivierung in der Wahrnehmung eher statischer Natur, da vor allem Wahrnehmungs- und Schmerzschwellen verändert sind, nicht aber die dynamische Veränderung der Schmerzwahrnehmung, wie bei der subjektiven Sensibilisierung bei chronisch muskuloskelettalen Beschwerden. 
Windup erklärt zeitliche Summation und Sensibilisierung, nicht aber chronischen Schmerz Nach einer intensiven Forschungsperiode in den 90er Jahren, in der für windup-Mechanismen eine zentrale Rolle beim chronischen Schmerz vermutet wurde, wird diese Ansicht derzeit in der Forschungsliteratur kritisch beurteilt. Windup ist kurzfristig und reversibel, er stellt deshalb kein dauerhaft wirksames Schmerzgedächtnis dar, welches persistierende oder chronisch wiederkehrende Schmerzen erklären könnte. Der windupMechanismus wird auch als aktivierungsabhängige Neuroplastizität betrachtet, im Gegensatz zu längerfristigen, aber reversiblen Modulationen der Nervenfunktion im Minuten bis Stundenbereich und zu irreversiblen strukturellen Modifikationen des Nervensystems im Zeitbereich von Tagen bis Jahren (15). Der zuletzt genannte Mechanismus der zentralen Sensitivierung wäre eher als neurobiologisches Modell der
Chronifizierung geeignet als der kurzfristige windup. Wie aber ist der Befund verstärkter Sensibilisierung dann bei chronischen Schmerzen zu erklären? Gibt es Erklärungsmodelle, die über die Interpretation der subjektiven Sensibilisierung als durch windup verursachte zeitliche Summation hinausgehen? Möglich wäre, dass die mutmaßlich durch windup verursachte subjektive Sensibilisierung mit den längerfristigen Prozessen einer zentralen Sensitivierung, wie der Langzeitpotenzierung (LTP), zusammenhängt. In diesem Sinne wäre ein veränderter windup nur ein Indikator für eine vorhandene LTP. Leider gibt es nur sehr spärliche Belege für diese These (11). Umgekehrt kann windup zwar Merkmale einer zentralen Sensitivierung (LTP) hervorrufen, wie Vergrößerung der rezeptiven Felder im Rückenmark und verstärkte Reaktionen auf afferente nozizeptive Aktivität (11). Dies reicht aber nicht aus, um windup als vermittelnden Prozess für langfristige Veränderungen

\section{Abb. 3 Die Bedeutung von Ängstlichkeit und Furcht-Vermei- dung in der Vorhersage der subjektiven Sensibilisierung bei chronischem Rückenschmerz}

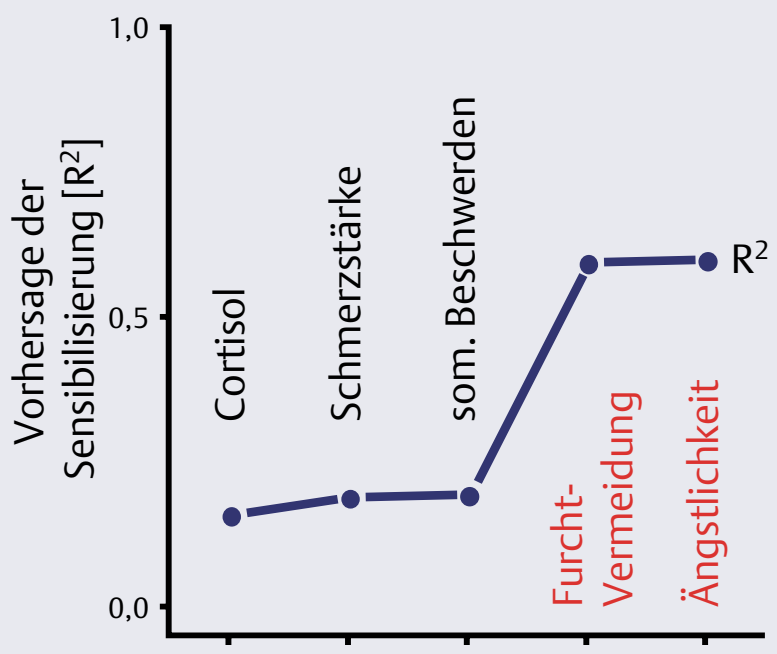

Vorhersage der subjektiven Sensibilisierung gegenüber tonischer Kontakthitzereizung (vgl. Abb. 1) auf der Basis eines multiplen Regressionsmodells bei Patienten mit chronischen unspezifischen Rückenschmerzen $(\mathrm{N}=36)$. Während biologische Parameter der Stressbelastung (Cortisol-Aufwachreaktion), Stärke der klinischen Schmerzen und somatische Komorbidität nur geringen Vorhersagewert für die Schmerzsensibilisierung besitzen, erklären dispositionelle Ängstlichkeit und schmerzbezogene Furcht-Vermeidung (fearavoidance-beliefs-questionnaire, FABQ; Pfingsten et al., 1997) erhebliche Varianzanteile der Sensibilisierungsreaktion $\left(R^{2}=\right.$ Determinationskoeffizient; der Anteil erklärter Varianz).

des Schmerzgedächtnisses zu beurteilen. Es ist wahrscheinlicher, dass weitere zentralnervöse Schmerzverarbeitungsprozesse auf die windupinduzierten subjektiven Empfindungsänderungen aufsetzen und eine weitere Verstärkung des windup im Gehirn stattfindet. In diesem Sinne ist es sogar denkbar, dass der spinale windup bei Gesunden und chronisch Schmerzkranken gar nicht unterschiedlich ausgeprägt ist, sondern vor allem die nachgeschaltete zerebrale Verarbeitung im Sinne des Schmerzgedächtnisses, hier in erster Linie basierend auf klassischer und operanter Konditionierung.

\section{Sensibilisierung, operante Konditionierung und Furcht- Vermeidung}

Verhaltensmedizinische Modelle nehmen an, dass die externen, sozialen Konsequenzen von offenem, expliziten Schmerzverhalten wie Klagen, Inanspruchnahme medizinischer und kompensatorischer Leistungen etc. maßgeblich zur Chronifizierung beitragen. Eine Reihe von experimentellen Studien hat die operante Modifizierbarkeit psychophysiologischer Schmerzreaktionen gezeigt (vgl. Beitrag von Flor in diesem Heft). Bei Studien mit Gesunden wurde eine damit einhergehende Wirkung auf den Schmerzbericht festgestellt. Es ist aber bis heute nicht klar, ob und wie operantes Lernen dabei auf die Schmerzwahrnehmung selbst wirkt.

Das Furcht-Vermeidungs-Modell von Lethem und Mitarbeitern $(8,13)$ geht demgegenüber davon aus, dass die unmittelbaren, in der Person wirkenden Folgen des Schmerzverhaltens, insbesondere die verstärkende Wirkung einer Schmerzreduktion, für die Chronifizierung wichtiger sind als externe soziale Konsequenzen. Die Furcht vor erneuten Schmerzen und ihre Reduktion durch antizipatorische Verhaltensweisen spielt dabei eine zentrale Rolle (1). Sie führt mit der Zeit zur Vermeidung immer geringerer nozizeptiver Reizintensitäten, beispielsweise durch Schonhaltung und Rückzug und wird durch intermittierende, oft harmlose Schmerzepisoden aufrechterhalten bzw. zusätz- 
lich gestärkt. Solche kurzfristigen geringen Zunahmen der Schmerzintensität durch ungünstige Haltung, Bewegungen usw., und vor allem ihre Steigerung durch dynamische Vorgänge wie windup und verwandte reversible Sensibilisierungsvorgänge, sind geeignet, die erforderlichen diskriminativen Signale zu liefern, von denen das Lernen übertriebener Schmerzvermeidung seinen Ausgang nehmen könnte.

Durch diesen diskriminativen Lernprozess kommt es zu einer „sensorischen Dekalibrierung“, so dass schließlich früher durchaus tolerable, im Extremfall sogar nichtschmerzhafte nozizeptive Reizstärken als schmerzhaft und interventionsbedürftig interpretiert werden (8). Das Furcht-Vermeidungs-Modell hat den Vorteil, sich nicht ausschließlich auf die indirekte Wirkung externer Verstärkungen offenen Schmerzverhaltens zu stützen, die nicht immer nachzuweisen sind. Stattdessen wird ein der Schmerzverarbeitung inhärenter Verstärkungsmechanismus angenommen, der unabhängig von äußeren Bedingungen und auslösenden Noxen einen schleichenden operanten Lernprozess („shaping“) zunehmender Schmerzempfindlichkeit in Gang setzen kann. Dieser Prozess muss nicht bewusst kontrolliert sein, sondern kann auch automatisch durch implizites Lernen ablaufen, bei dem die Betroffenen entweder die unterschwelligen sensorischen Auslöser nicht bewusst bemerken oder sich der wirksamen Verstärkung und des eigenen Flucht- und Vermeidungsverhaltens nicht bewusst sein müssen.

Es ist sogar zu vermuten, dass Schmerzen in Folge zeitlich begrenzter Noxen mit klarem Beginn der Schmerzen und Ende bei erfolgreichem Eingriff eine geringere $\mathrm{Ge}$ fahr der schleichenden „sensorischen Dekalibration“ mit sich bringen als oft diffuse, schlecht lokalisierbare Schmerzen unklarer Ursache, bei denen die Diskrimination des zugrunde liegenden nozizeptiven Signals schwer fällt. Der Lernmechanismus spielt daher nach unserer Ansicht gerade bei solchen chronischen Schmerzsyndromen,

Abb. 4 Funktionelle Bildgebung der Sensibilisierungswahrnehmung bei Fibromyalgie (,percept-related fMRI”)
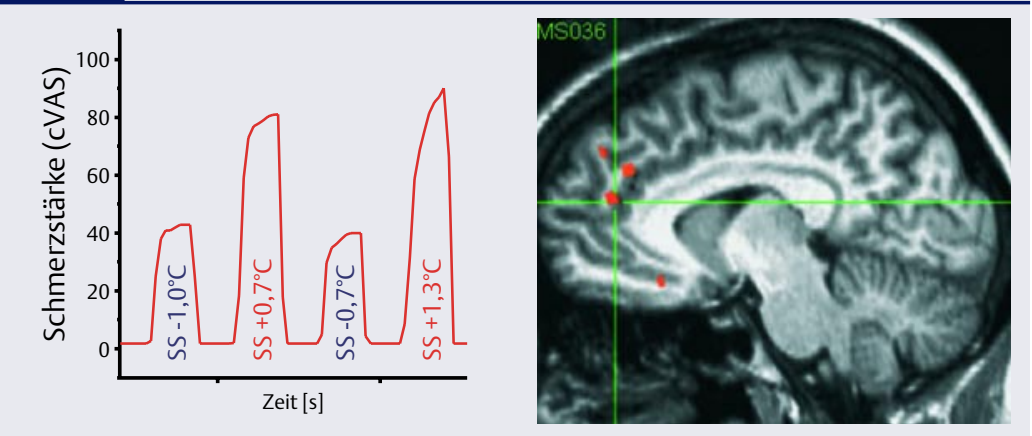

Bei funktionellen MR-Messungen wird Sensibilisierung im Verlauf tonischer Hitzereize von $35 \mathrm{sec}$. Dauer mit einer kontinuierlichen Einschätzung auf einer visuellen Analogskala (continuous VAS; cVAS) gemessen (A). Die Reizstärken werden relativ zur individuellen Schmerzschwelle (SS) definiert. Der Verlauf der cVAS zeigt bei tonischer konstanter Reizung eine Zunahme der Empfindungsstärke bei allen Reizstärken, wie es typischerweise bei Fibromyalgiepatienten gefunden wird. Diese Sensibilisierung kann durch eine Regressionsanalyse in der funktionellen MR-Bildgebung als Aktivierung im Gehirn sichtbar gemacht werden (B): Vor allem Teile des anterioren und perigenualen Gyrus cinguli, die mit affektiver Reizverarbeitung assoziiert sind, sind bei Sensibilisierung aktiv (MR-Bildgebung in Kooperation mit Dr. Michael Amann, Deutsches Krebsforschungszentrum Heidelberg).

bei denen umrissene Noxen zu Beginn der chronischen Entwicklung in der Regel nicht bekannt sind, d.h. den funktionellen somatischen und viszeralen Schmerzsyndromen, am ehesten eine bedeutende Rolle in Pathogenese und Chronifizierung. Damit liefert das Modell zumindest theoretisch einen verhaltenspsychologischen Ansatz zur Erklärung der progressiven Veränderung der Schmerzwahrnehmung bei chronischen Patienten.

Sensibilisierung, operante Konditionierung und Furcht-Vermeidung: erste empirische Befunde

Kann operante Konditionierung subjektive Sensibilisierung verändern bzw. verstärken? Wir prüften dies in einem Experiment mit Gesunden, bei dem wir versuchten, selektiv entweder Sensibilisierung oder Habituation durch operantes Lernen zu verstärken.

Es konnte gezeigt werden, dass Sensibilisierung und Schmerzwahrnehmung tonischer Hitzereize bei Gesunden im Verlauf einer Stunde durch kontingente (unmittelbar auf das Verhalten folgende) Erhöhung (Bestrafung: ein aversiver Reiz wird gegeben) oder Erniedrigung (nega- tive Verstärkung: ein aversiver Reiz bleibt aus) der Reizstärke verändert werden können (5). Eine neuere Untersuchung aus unserer Gruppe bestätigt diesen Befund bei Gesunden.

Derzeit wird in weiteren Studien geprüft, inwieweit dies für chronisch Schmerzkranke replizierbar ist und ob Unterschiede in der operanten Konditionierbarkeit zwischen Gesunden und Schmerzpatienten bestehen. Aus dem FurchtVermeidungsmodell der operanten Schmerzverstärkung lassen sich weiterhin Hypothesen über die pathogenetische Bedeutung der Komorbidität chronischer Schmerzen mit Angst, Depression und somatoformen Störungen ableiten. Dazu gehört insbesondere die Annahme, dass schmerzbezogene Angst und komorbide Schmerzen zum einen operante Verstärkungsmechanismen erleichtern und zum anderen eine Löschung operanten Schmerzverhaltens durch schmerzbezogene Angst erschweren (1).

Ähnliches gilt für die regelmäßig, wenn auch uneinheitlich berichteten Auffälligkeiten der endokrinen und autonomen Stressreaktion. Erste Daten aus einer Querschnittstudie un- 
serer Gruppe bei chronischen unspezifischen Rückenschmerzen zeigen, dass in der Tat ein Zusammenhang zwischen der verstärkten Sensibilisierung der Rückenschmerzpatienten und der Disposition zu Angst und vor allem zur Angst-Vermeidung bei diesen Patienten besteht (Abb. 3). Dies könnte eine erhöhte Vulnerabilität dieser Patienten für operantes Lernen von Schmerzsensibilisierung anzeigen und für eine nachfolgende sensorische Dekalibrierung, bei der mit der Zeit immer geringere nozizeptive Signale eine erhöhte Schmerzempfindung auslösen können.

Ferner prüfen wir derzeit in funktionellen Bildgebungsstudien, ob die verstärkte Sensibilisierung bei chronisch Schmerzkranken entsprechende Veränderungen in der zerebralen funktionellen Aktivierung auf tonische Hitzereize bewirkt (Abb. 4). Erste Ergebnisse unserer Gruppe und anderer Arbeitsgruppen zeigen bei Fibromyalgiepatienten eine erhöhte zerebrale Aktivierung im Vergleich zu Gesunden, bei geringeren objektiven Reizstärken. Unsere derzeitigen Befunde belegen außerdem, dass Sensibilisierung im Gehirn nachweisbar ist und dort vor allem mit affektiven Funktionen, wie Furcht und Furcht-Vermeidung assoziiert ist (Abb. 4).

\section{- Fazit}

Schmerzsensibilisierung ist vor allem bei Patienten mit chronischen muskuloskelettalen Schmerzen unklarer Ätiologie ein häufiges klinisches Merkmal, welches mit überdauernden Veränderungen in der nozizeptiven Signalverarbeitung assoziiert ist. Prozesse der aktivierungsabhängigen Neuroplastizität an spinalen Hinterhornneuronen (windup) sind allerdings nicht geeignet, die zeitlich überdauernden Merkmale klinischer Schmerzen und Chronifizierung von Schmerz zu erklären. Operantes Lernen verstärkter Sensibilisierung in Verbindung mit einer spezifischen Vulnerabilität der betroffenen Patienten durch Ängstlichkeit und Furcht-Vermeidungsverhalten gegenüber Schmerzen könnten die Ursache sein für eine sensorische Dekalibrierung der Schmerzwahr- nehmung, wie sie von der FurchtVermeidungshypothese postuliert wird. Erste empirische Befunde belegen die Konditionierbarkeit der Schmerzsensibilisierung bei Gesunden und den Zusammenhang verstärkter Sensibilisierung mit dispositioneller Angst und Furcht-Vermeidung bei chronisch Schmerzkranken. Verstärkte Sensibilisierung chronischer Patienten ist außerdem in der kortikalen Aktivierung nachweisbar, vor allem in Regionen, die mit der affektiven Reizverarbeitung, Furcht und Vermeidungsverhalten, assoziiert sind.

\section{Pain memory and sensitization} Chronic pain patients frequently experience an increase of perceived pain intensity during constant repetitive or tonic stimulation with experimental pain applied for the purpose of psychophysical pain diagnostics. Such a sensitization of pain is regarded as a perceptible correlate of neuronal plasticity on early peripheral and spinal stages of pain processing and might be an indicator for longterm changes in afferent pain processing, due to the process of pain becoming chronic. Perceptual sensitization however, is modulated not only by subcortical neuroplasticity, but rather also through cerebral learning processes like classical and operant conditioning. Operant learning of increasing sensitization in connection with the psychological mechanism of fear-avoidance is possibly a central cause of chronic pain of unknown etiology, especially in pain syndromes with impairment of the musculoskeletal system.

\section{Key Words}

Chronic pain - pain memory perceptual sensitization - psychophysics - fear-avoidance - operant learning

\section{Literatur}

1. Al-Obaidi SM, Nelson RM, Al-Awadhi S, Al-Shuwaie N. The role of anticipation and fear of pain in the persistence of avoidance behavior in patients with chronic low back pain. Spine 2000; 25: 1126-1131

2. Eide PK. Wind-up and the NMDA receptor complex from a clinical perspective. European Journal of Pain 2000; 4: 5-17

3. Graven-Nielsen T, Aspegren Kendall S,
Henriksson KG, Bengtsson M, Sorensen J, Johnson A, et al. Ketamine reduces muscle pain, temporal summation, and referred pain in fibromyalgia patients. Pain 2000; 85 : 483-491

4. Klein T, Magerl W, Hopf HC, Sandkuhler J, Treede RD. Perceptual correlates of nociceptive long-term potentiation and longterm depression in humans. J Neurosci 2004; 24: 964-971

5. Kleinböhl D, van Ackern K, Hölzl R, Huse E, Möltner A, Wöhrle C. Operant conditioning of pain perception in an experimental tonic heat pain model. Proceedings of the $8^{\text {th }}$ World Congress on Pain. Seattle: IASP Press, 1996; 477

6. Kleinböhl D, Hölzl R, Möltner A, Rommel C, Osswald P-M, Weber C. Psychophysical measures of sensitization to tonic heat discriminate chronic pain patients. Pain 1999; 81: 35-43

7. Kleinböhl D, Amann M, Hölzl R, Möltner A, Schad LR. Psychophysics and functional imaging of sensitization in healthy subjects and chronic musculosceletal pain syndromes. J Psychophysiol 2003; 17: 161

8. Lethem J, Slade PD, Troup JDG, Bentley G. Outline of a fear-avoidance model of exaggerated pain perception - I. Behav Res Ther 1983; 21: 401-408

9. Maixner W, Fillingim R, Sigurdsson A, Kincaid S, Silva S. Sensitivity of patients with painful temporomandibular disorders to experimentally evoked pain: evidence for altered temporal summation of pain. Pain 1998; 76: 71-81

10. Mense S. Pathophysiologie des Rückenschmerzes und seine Chronifizierung. Der Schmerz 2001; 6: 413-418

11. Sandkühler J. Learning and memory in pain pathways. Pain 2000; 88: 113-118

12. Staud R, Vierck C], Cannon RL, Mauderli AP, Price DD. Abnormal sensitization and temporal summation of second pain (windup) in patients with fibromyalgia syndrome. Pain 2001; 91: 165-175

13. Vlaeyen JW, Linton SJ. Fear-avoidance and its consequences in chronic musculoskeletal pain: a state of the art. Pain 2000; 85: 317-332

14. Weissman-Fogel I, Sprecher E, Granovsky Y, Yarnitsky D. Repeated noxious stimulation of the skin enhances cutaneous pain perception of migraine patients in-between attacks: clinical evidence for continuous sub-threshold increase in membrane excitability of central trigeminovascular neurons. Pain 2003; 104: 693-700

15. Woolf CJ, Salter MW. Neuronal plasticity: increasing the gain in pain. Science 2000; 288: 1765-1769

\author{
Korrespondenzadresse: \\ Dr. phil. Dieter Kleinböhl \\ Universität Mannheim \\ Otto-Selz-Institut \\ Labor für Klinische Psychophysiologie \\ Schloss EW 184 \\ 68131 Mannheim \\ kleinboehl@osi.uni-mannheim.de
}

